\title{
Production of reactive persulfide species in chronic obstructive pulmonary disease
}

\author{
Tadahisa Numakura, ${ }_{1}^{1}$ Hisatoshi Sugiura, ${ }_{1}^{1}$ Takaaki Akaike, ${ }^{2}$ \\ Tomoaki Ida, ${ }^{2}$ Shigemoto Fujii, ${ }^{2}$ Akira Koarai, ${ }^{1}$ Mitsuhiro Yamada, ${ }^{1}$ Katsuhiro Onodera, ${ }^{1}$ \\ Yuichiro Hashimoto, ${ }^{1}$ Rie Tanaka, ${ }^{1}$ Kei Sato, ${ }^{1}$ Yutaka Shishikura, ${ }^{1}$ Taizou Hirano, ${ }^{1}$ \\ Satoru Yanagisawa, ${ }^{1}$ Naoya Fujino, ${ }^{1}$ Tatsuma Okazaki, ${ }^{1}$ Tsutomu Tamada, \\ Yasushi Hoshikawa, ${ }^{3}$ Yoshinori Okada, ${ }^{4}$ Masakazu Ichinose ${ }^{1}$
}

\begin{abstract}
- Additional material is published online only. To view please visit the journal online (http://dx.doi.org/10.1136/ thoraxjnl-2016-209359).
\end{abstract}

'Department of Respiratory Medicine, Tohoku University Graduate School of Medicine, Sendai, Japan

${ }^{2}$ Department of Environmental Health Sciences and Molecular Toxicology, Tohoku University Graduate School of Medicine, Sendai, Japan

${ }^{3}$ Department of Thoracic Surgery, Fujita Health University School of Medicine, Toyoake, Japan

${ }^{4}$ Department of Thoracic Surgery, Institute of Development, Aging and Cancer, Tohoku University, Sendai, Japan

\section{Correspondence to}

Dr Hisatoshi Sugiura, Department of Respiratory Medicine, Tohoku University Graduate School of Medicine, Seiryou-machi, Aoba-ku, Sendai 980-8574, Japan; sugiura@rm. med.tohoku.ac.jp

Received 28 August 2016 Revised 15 May 2017 Accepted 5 June 2017

Published Online First 19 July 2017

\section{Linked}

- http://dx.doi.org/10.1136/ thoraxjnl-2017-210703

\section{CrossMark}

To cite: Numakura $T$ Sugiura H, Akaike T, et al.

Thorax 2017;72:1074-1083.

\section{ABSTRACT}

Background Oxidative stress is a major aetiological

factor driving chronic obstructive pulmonary disease (COPD). Recently recognised as potent antioxidants, reactive persulfide and polysulfide species are biosynthesised by cystathionine $\beta$-synthase and cystathionine $\gamma$-lyase. The production of reactive persulfide and polysulfide species in the lungs of patients with COPD remain unknown.

Objectives The aim of this study was to examine the production of reactive persulfides and polysulfides, such as glutathione persulfide (GSSH), cysteine persulfide (CysSSH) and glutathione trisulfide (GSSSH), in lungresident cells and epithelial lining fluid (ELF) obtained from patients with mild to moderate COPD.

Methods Lung tissues, primary lung cells, ELF and sputum were obtained. The amounts of reactive persulfides and polysulfides in the cells and ELF were measured by liquid chromatography-tandem mass spectrometry with $\beta$-(4-hydroxyphenyl) ethyl iodoacetamide as a trapping agent for hydroper/ polysulfides. The amounts of synthases in the lung tissues, sputum and primary cells were quantified. Results The amounts of GSSH, CysSSH and GSSSH were decreased in the lung cells and ELF from patients with COPD. The amounts of reactive persulfides and polysulfides in the lung cells had a positive correlation with the degree of airflow limitation. By contrast, the amounts of the synthases were increased in the lung tissues and sputum cells of patients with COPD.

Conclusions We have identified a decrease in reactive persulfide and polysulfide species in the lungs of patients with COPD. These data suggest that the newly detected antioxidants reactive persulfides and polysulfides could be associated with the redox balance in the lungs of patients with COPD.

\section{INTRODUCTION}

The major aetiological factor driving chronic obstructive pulmonary disease (COPD) is oxidative stress in the lung. ${ }^{1-6}$ Oxidative stress arises from endogenous antioxidant defences that are genetically impaired and/or are overwhelmed by the presence of reactive oxygen species (ROS) and reactive nitrogen species (RNS), which are both exogenously and endogenously derived. ${ }^{2-10}$ Although glutathione (GSH) is believed to provide a primary

\section{Key messages}

What is the key question?

- Do reactive persulfide and polysulfide species, newly identified as potent antioxidants, exist in human lungs, and if so, do they have a role in the redox status of chronic obstructive pulmonary disease (COPD)?

What is the bottom line?

- This study is the first to quantify the amounts of reactive persulfides and polysulfides (eg, glutathione persulfide, cysteine persulfide and glutathione trisulfide) in human lungs, and the amounts of reactive persulfide and polysulfide species are decreased in the lung-resident cells as well as in airway epithelial lining fluid obtained from patients with COPD.

Why read on?

- These data suggest that a decrease in reactive persulfides and polysulfides is associated with a redox imbalance in the lungs of patients with COPD. Therefore, targeting reactive persulfides and polysulfides may be a potential new strategy to combat various pathogenic processes mediated by oxidative stress in COPD.

antioxidant defence against oxidative stress, the profiles of endogenous antioxidants in the lung have not yet been fully elucidated. ${ }^{311} 12$

Recently, we originally reported a liquid chromatography electrospray ionisation tandem mass spectrometry (LC-ESI-MS/MS) based method using monobromobimane (MBB) to measure reactive persulfide and polysulfide species, such as glutathione persulfide (GSSH), cysteine persulfide (CysSSH) and glutathione trisulfide (GSSSH), in cells and plasma from humans. ${ }^{13}{ }^{14}$ We also demonstrated that reactive persulfides are highly reactive and extremely powerful antioxidants that actually regulate oxidative stress and redox signalling mediated by various electrophilic oxidants. ${ }^{13-19}$ Additionally, we showed that CysSSH is biosynthesised from cystine by cystathionine $\beta$-synthase (CBS) and cystathionine $\gamma$-lyase (CSE), which in turn may contribute to the production of GSSH and other 
CysSSH derivatives formed in humans. ${ }^{14} 1518$ The antioxidant capacity and reactivity of GSSH and CysSSH are approximately 10-100 times greater than those of GSH and cysteine (CysSH). ${ }^{14}$ 151719 Currently, the amounts of reactive persulfide and polysulfide species and their biosynthesis in the lungs of patients with COPD remain unknown. The goal of this study was to rigorously identify reactive persulfide and polysulfide species generated under various physiological and pathophysiological conditions. However, the LC-MS/MS analysis with MBB that was employed previously had some disadvantages; MBB degrades the polysulfide structure because of its strong electrophilicity, which causes an artefactual decrease in various polysulfides and their $\mathrm{MBB}$ adducts. It is therefore essential to use a more effective trapping agent, that is $\beta$-(4-hydroxyphenyl)ethyl iodoacetamide (HPE-IAM), which can effectively react with hydropolysulfides without affecting the entire polysulfide structure and can form more stable adducts than those created in the MBB reaction.

The present study had the following aims: to determine reactive persulfide and polysulfide amounts generated in primary lung-resident cells and epithelial lining fluid (ELF) from patients with COPD using LC-ESI-MS/MS with a new per/polysulfide probe (ie, HPE-IAM); and to analyse the expression of CBS and CSE in the lungs and airways of patients with COPD.

\section{MATERIALS AND METHODS}

A complete version of the materials and methods is provided in the online supplementary material.

\section{Subjects}

Study subjects from Tohoku University Hospital participated in the study between January 2013 and July 2016. The subjects for each study using primary cultured cells, ELF samples, histological materials or sputum samples were independently recruited and did not overlap. All patients with COPD satisfied the Global Initiative for Chronic Obstructive Lung Disease (GOLD) guideline criteria. ${ }^{1}$ Current smokers were excluded, and all ex-smokers had quit smoking for at least 1 year before the study. Thirteen patients with COPD and 17 non-COPD subjects were included in the primary cell study (table 1). Both bronchus and peripheral lung tissues were obtained from patients with or without COPD undergoing lung cancer surgery, avoiding areas involved by tumours. The tissues were used for the culture of primary lung-resident cells, including bronchial epithelial cells and lung fibroblasts. ${ }^{2021}$ Thirteen patients with COPD and 10 non-COPD subjects were included in the ELF study (see online supplementary table S1). ELF samples were obtained by a bronchoscopic microsampling method described in a previous study. ${ }^{22}$ Eighteen patients with COPD and 32 non-COPD subjects (18 non-COPD never-smokers and 14 non-COPD ex-smokers) were included in the immunohistochemical study (see online supplementary table S2). The lung tissues obtained from patients with or without COPD undergoing lung cancer surgery were used for immunohistochemical staining. Twenty-six patients with COPD and 11 non-COPD subjects were included in the sputum study (see online supplementary tables S3 and S4). Sputum samples were obtained by using a hypertonic saline inhalation method. ${ }^{9}$ All subjects performed pulmonary function tests after enrolment in the study. Written informed consent was obtained from all subjects who participated in this study. All experiments in this study were approved by the ethics committee of Tohoku University Graduate School of Medicine.

\section{Determination of reactive persulfide and polysulfide species}

Intracellular and extracellular reactive persulfide and polysulfide species were quantified by LC-MS/MS with HPE-IAM as a new trapping agent for hydropersulfides and polysulfides (see online supplementary figure S1). Compared with the MBB trapping reported earlier, the use of HPE-IAM greatly improved the efficacy of capture of the various persulfides and polysulfides and could stabilise their respective HPE-IAM adducts (see online supplementary figure S1). To measure the amounts of intracellular reactive persulfides of primary cells under basal conditions, primary bronchial epithelial cells were seeded at a density of $1 \times 10^{5}$ cells $/ \mathrm{mL}$ in a 24 -well plate, cultured for 48 hours and then further cultured in growth factor-free medium for the next 24 hours. The cultured cells were lysed by sonication in

Table 1 Characteristics of subjects in the primary cell study

\begin{tabular}{|c|c|c|c|c|c|}
\hline \multirow[b]{2}{*}{ Subjects (n) } & \multicolumn{2}{|c|}{ Non-COPD } & \multicolumn{2}{|l|}{ COPD } & \multirow[b]{2}{*}{$\mathrm{p}$ Value } \\
\hline & 17 & & 13 & & \\
\hline Age (years) & 67.0 & $(62.5-75.0)$ & 68.0 & $(65.5-73.5)$ & 0.642 \\
\hline Ex-smokers/never-smokers & $6 / 11$ & & $13 / 0$ & & $<0.001$ \\
\hline Smoking history (pack-year) & 0.5 & $(0.0-29.6)$ & 55 & $(37.0-69.0)$ & $<0.001$ \\
\hline $\mathrm{FEV}_{1}(\mathrm{~L})$ & 2.34 & $(2.01-2.79)$ & 2.27 & $(1.85-2.48)$ & 0.425 \\
\hline $\mathrm{FEV}_{1} \%$ predicted & 103.7 & $(94.9-114.9)$ & 79.4 & $(69.2-92.3)$ & $<0.001$ \\
\hline $\mathrm{FEV}_{1} / \mathrm{FVC}(\%)$ & 80.4 & $(76.2-84.3)$ & 67.2 & $(56.2-68.4)$ & $<0.001$ \\
\hline$D_{\text {LCO }} \%$ predicted & 116.1 & (91.1-138.1) & 90.4 & $(72.0-105.3)$ & 0.009 \\
\hline
\end{tabular}

Data are presented as the median (with interquartile ranges). Data were analysed using a Wilcoxon rank sum test or Fisher's exact test.

$\mathrm{D}_{\text {Lco' }}$ diffusing capacity of the lung for carbon monoxide; $\mathrm{FEV}_{1}$, forced expiratory volume in $1 \mathrm{~s}$; FVC, forced vital capacity; GOLD, Global Initiative for Chronic Obstructive

Pulmonary Disease; LABA, long-acting $\beta 2$ antagonist; LAMA, long-acting muscarinic antagonist; $\mathrm{V}_{\mathrm{A}^{\prime}}$ alveolar volume. 
a $0.2 \mathrm{~mL}$ cold methanol solution containing $1 \mathrm{mM}$ HPE-IAM. To measure the amounts of reactive persulfide and polysulfide species in ELF, $10 \mu \mathrm{L}$ of ELF was mixed with $100 \mu \mathrm{L}$ of cold methanol solution containing $1 \mathrm{mM}$ HPE-IAM. These cell lysates and ELF sample solutions were incubated at $37^{\circ} \mathrm{C}$ for $30 \mathrm{~min}$. After centrifugation $\left(14000 \times \mathrm{g}, 10 \mathrm{~min}, 4^{\circ} \mathrm{C}\right)$, the supernatants were collected. Aliquots of the supernatants were diluted 10-100 times with distilled water containing known amounts of stable isotope-labelled internal standards, as schematised in online supplementary figure S1. The LC-ESI-MS/ MS analyses were performed using a triple quadrupole (Q) mass spectrometer LCMS-8050 (Shimadzu, Kyoto, Japan) coupled to a Nexera UHPLC system (Shimadzu). Polysulfide derivatives were separated using a Nexera UHPLC with a YMC-Triart C18 column $(50 \times 2.0 \mathrm{~mm}$ inner diameter $)$ with a linear $5-90 \%$ methanol gradient for $15 \mathrm{~min}$ in $0.1 \%$ formic acid at $40^{\circ} \mathrm{C}$ (flow rate $0.2 \mathrm{~mL} / \mathrm{min}$; injection volume $10 \mu \mathrm{L}$ ). MS spectra were obtained with the temperature of the ESI probe, desolvation line, and heat block at $300^{\circ} \mathrm{C}, 250^{\circ} \mathrm{C}$ and $400^{\circ} \mathrm{C}$, respectively; the nebuliser, heating and drying nitrogen gas flows were set to 3, 10 and 10 $\mathrm{L} / \mathrm{min}$, respectively. Various polysulfide derivatives were identified and quantified using multiple reaction monitoring (MRM).

(A)
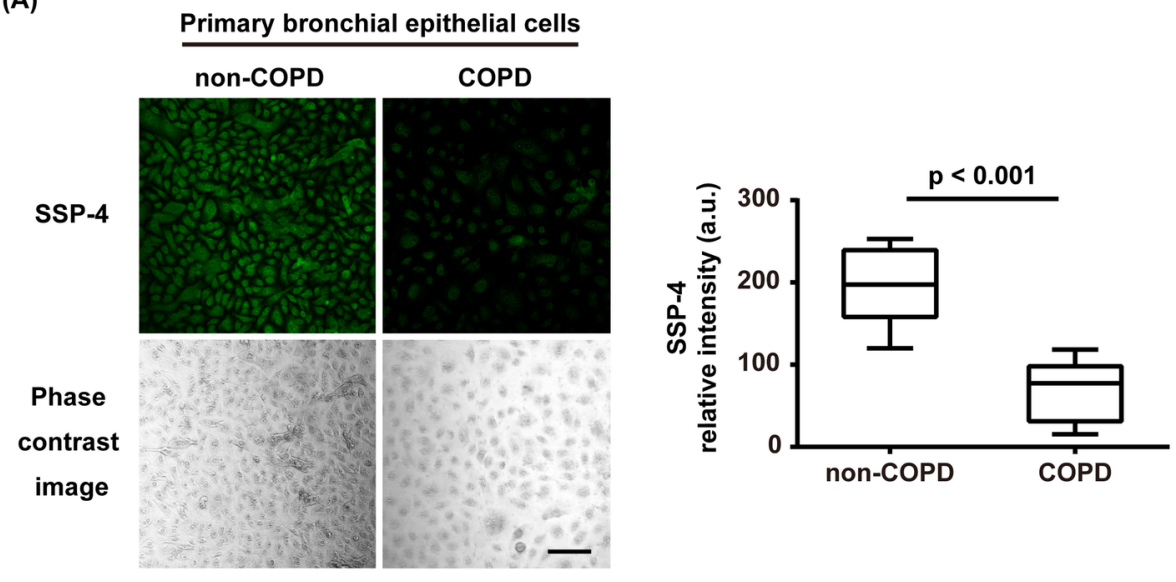

(B)
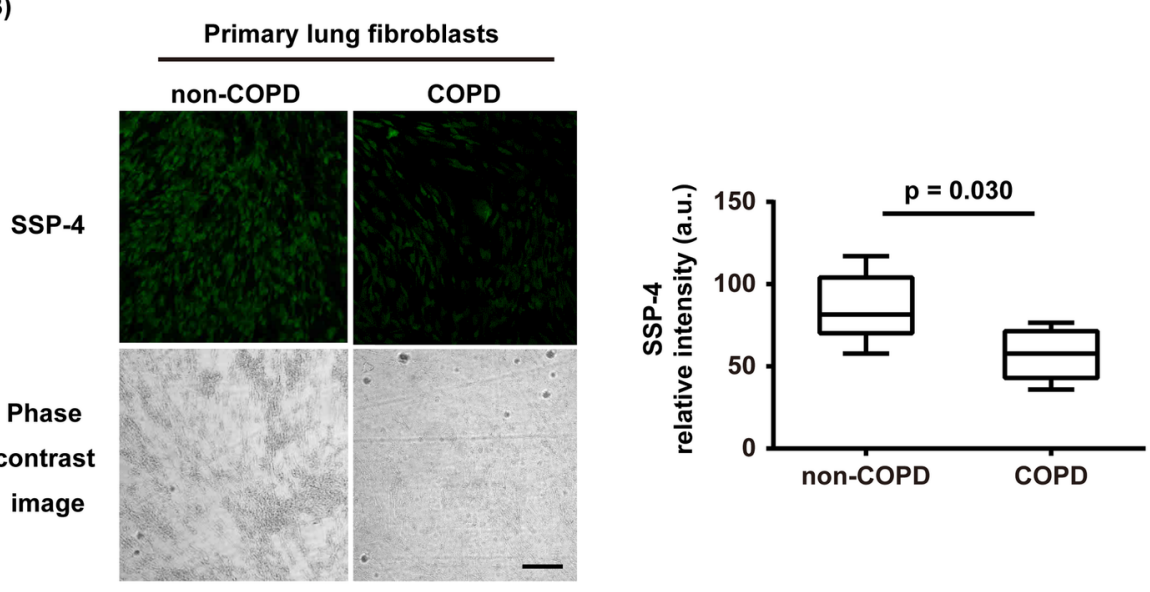

(C)

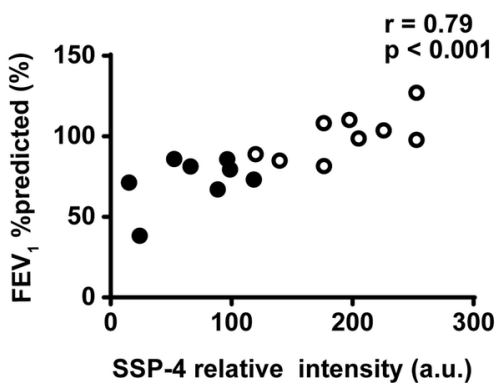

(D)

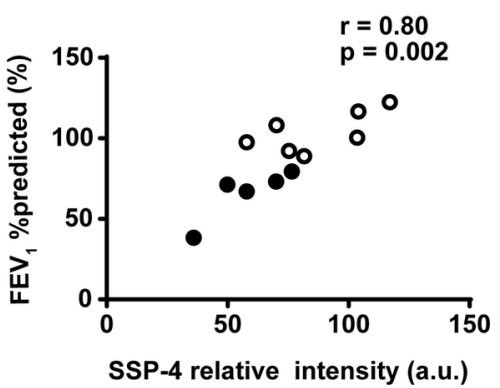

Figure 1 Decrease in intracellular reactive persulfide and polysulfide species in human lung-resident cells from patients with chronic obstructive pulmonary disease (COPD). Representative images of sulfane sulfur probe 4 (SSP-4) fluorescence in primary bronchial epithelial cells (A, left panels) and primary lung fibroblasts ( $B$, left panels) are shown. The amounts of intracellular reactive persulfides and polysulfides in primary bronchial epithelial cells ( $A$, right panel) and primary lung fibroblasts ( $B$, right panel) from study subjects were measured using a fluorescence microplate reader (bronchial epithelial cells: non-COPD, $n=9 ;$ COPD, $n=8$; lung fibroblasts: non-COPD, $n=7 ;$ COPD, $n=5$ ). Correlations between the percent predicted values of forced expiratory volume in $1 \mathrm{~s}$ (FEV $\%$ predicted) and the SSP-4 fluorescence intensities in primary bronchial epithelial cells (C) and primary lung fibroblasts (D) are shown. $r$ is the correlation coefficient. Data represent the median (with interquartile ranges). Data were analysed using Wilcoxon rank sum tests. Correlations were established using Spearman's tests. Scale bars $=100 \mu \mathrm{m}$. a.u., arbitrary units. 
See online Supplementary table S5 which summarises the MRM parameters for each HPE-IAM adduct of different per/polysulfides, including their stable isotope-labelled derivatives.

\section{Statistical analysis}

All data are expressed as medians (with interquartile ranges). Statistical tests were performed using GraphPad Prism 6.0J (GraphPad Software Inc, San Diego, California, USA) or R version 3.4.0 (R Foundation for Statistical Computing, Vienna, Austria). Statistical analyses were assessed by Wilcoxon rank sum tests to compare all continuous variables between two groups. For comparisons among three groups, a Kruskal-Wallis test was followed by Dunn's test. Statistical correlation analyses were performed using Spearman's test. Multiple regression analyses were performed for evaluating the effect of potential confounding factors. $p$ Values less than 0.05 were considered significant.

\section{RESULTS}

Decrease in intracellular reactive persulfide species in primary lung-resident cells from patients with COPD

To investigate the amounts of reactive persulfides and polysulfides in primary lung-resident cells, we used a sulfane sulfur probe 4 (SSP-4), which is a recently developed fluorescence probe with high specificity for reactive persulfides and polysulfides. The characteristics of the subjects in the primary cell study are provided in table 1. Staining with SSP-4 revealed that the amounts of intracellular reactive persulfides and polysulfides were significantly decreased in the bronchial epithelial cells from the patients with COPD (77.3 (31.3-98.2) arbitrary units (a.u.)) compared with those from the non-COPD subjects (197.4 (158.0-239.3) a.u., $\mathrm{p}<0.001$, figure $1 \mathrm{~A})$. Reactive persulfides and polysulfides were also decreased in primary lung fibroblasts (COPD, 57.9 (42.971.4) vs non-COPD, 81.6 (70.2-104.0) a.u., $\mathrm{p}=0.030$, figure $1 \mathrm{~B})$. The levels of SSP-4 fluorescence in primary bronchial epithelial cells $(\mathrm{r}=0.79, \mathrm{p}<0.001$, figure $1 \mathrm{C})$ as well as primary lung fibroblasts $(r=0.80, p=0.002$, figure $1 D)$ were significantly correlated with the degree of airflow limitation in the patients from which these cells were collected.

\section{Decrease in GSSH and CysSSH in primary bronchial epithelial} cells from patients with COPD

Next, we measured the amounts of individual reactive persulfide species in primary cells by LC-ESI-MS/MS (figure 2 and see online supplementary figure S2). The amounts of intracellular GSSH were significantly decreased in bronchial epithelial cells from patients with $\operatorname{COPD}(50.5$ (40.1-62.1) nmol/g protein) compared with those from non-COPD subjects $(70.9$ (62.7-108.7) nmol/g protein, $\mathrm{p}=0.008$, figure $2 \mathrm{~A}$ ). Similarly, the amounts of intracellular CysSSH were significantly decreased in bronchial epithelial cells from patients with COPD (20.5 (16.0-23.5) nmol/g protein) compared with those from non-COPD subjects (30.9 (22.9$38.7) \mathrm{nmol} / \mathrm{g}$ protein, $\mathrm{p}=0.007$, figure $2 \mathrm{~B}$ ). Interestingly, there was no significant difference in the amounts of GSH in the bronchial epithelial cells between the COPD patients (46.5 (29.9-73.7) $\mu \mathrm{mol} / \mathrm{g}$ protein) and non-COPD subjects $(60.3$ (45.8-72.9) $\mu \mathrm{mol} / \mathrm{g}$ protein, $\mathrm{p}=0.208$, figure $2 \mathrm{C}$ ) as well as the amounts of $\mathrm{CysSH}$ between the two groups (COPD, 4.8 (3.9-6.3) vs non-COPD, 6.1 $(5.7-8.7) \mu \mathrm{mol} / \mathrm{g}$ protein, $\mathrm{p}=0.070$, figure $2 \mathrm{D})$.

\section{Increased production of reactive oxygen species and proinflammatory cytokines and chemokines in primary lung- resident cells from patients with COPD}

We further examined the generation of reactive oxygen species (ROS) in the primary cells from the study subjects (table 1). The amounts of ROS were significantly increased in bronchial epithelial cells from patients with COPD (27.6 (25.2-30.7) a.u.) compared with those from the non-COPD subjects $(12.8$

(B)

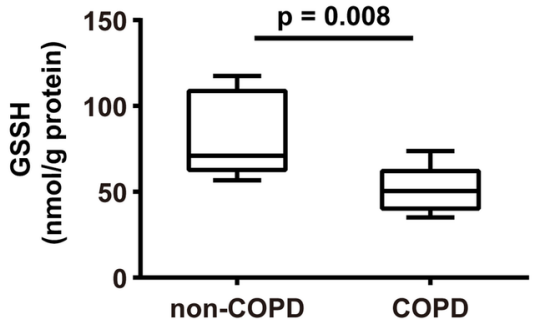

(C)

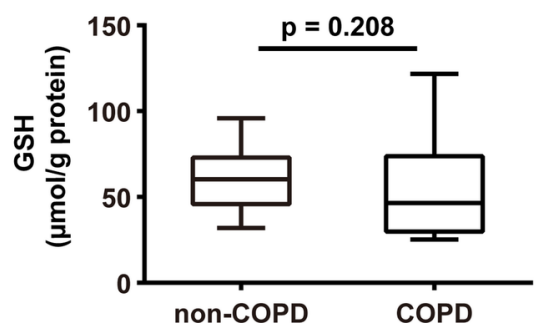

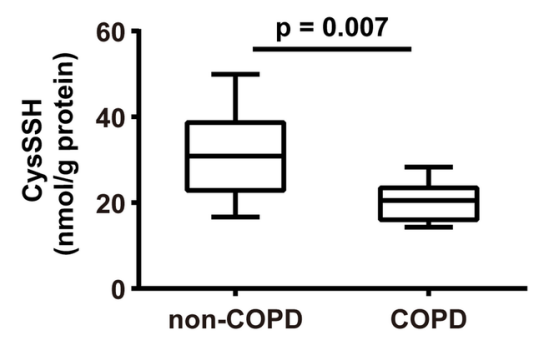

(D)

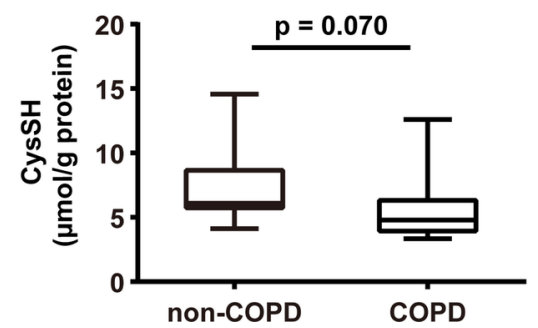

Figure 2 Determination of intracellular reactive persulfide species in primary bronchial epithelial cells. Cell lysates of primary bronchial epithelial cells from patients with chronic obstructive pulmonary disease (COPD) and non-COPD subjects were subjected to liquid chromatography (LC)-tandem mass spectrometry (MS)/MS. Intracellular glutathione persulfide (GSSH) (non-COPD, $n=9 ; C O P D, n=7)(A)$, cysteine persulfide (CysSSH) (non-COPD, $n=12 ; C O P D, n=8)(B)$, glutathione (GSH) (non-COPD, $n=14 ; C O P D, n=10)(C)$ and cysteine (CysSH) (non-COPD, $n=13 ; C O P D, n=9)(D)$ were identified. Data represent the median (with interquartile ranges). Data were analysed using Wilcoxon rank sum tests. 
(10.0-16.8) a.u., $\mathrm{p}=0.029$, figure $3 \mathrm{~A}$ and $\mathrm{B})$ as well as in lung fibroblasts between the two groups (COPD, 20.6 (19.1-26.4) vs non-COPD, 15.6 (7.1-17.4) a.u., $\mathrm{p}=0.015$, figure $3 \mathrm{C}$ and D). The amounts of interleukin (IL) -8 were also significantly increased in the cultured media of the bronchial epithelial cells from patients with COPD (480.6 (229.0-611.4) pg/mL) compared with those from non-COPD subjects under basal conditions (187.6 (145.2-236.9) pg/mL, p=0.030, figure 3E). Similarly, the amounts of IL-1 $\beta$ (figure $3 F$ ), IL-6 (figure $3 \mathrm{G}$ ) and monocyte chemotactic protein $(\mathrm{MCP})-1$ (figure $3 \mathrm{H}$ ) were significantly increased in the cultured media of bronchial epithelial cells from patients with COPD (IL-1 $\beta$ : COPD, 19.1 $(11.8-23.5)$ vs non-COPD, $3.3(0.6-7.0) \mathrm{pg} / \mathrm{mL}, \mathrm{p}=0.016$; IL-6: COPD, $52.4(21.4-85.2)$ vs non-COPD subjects, 2.2

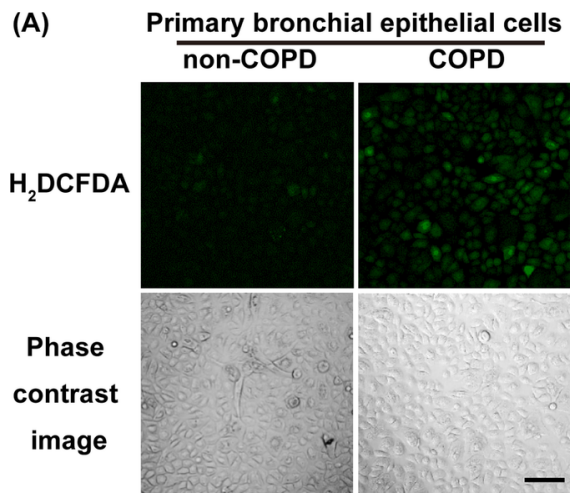

(C)

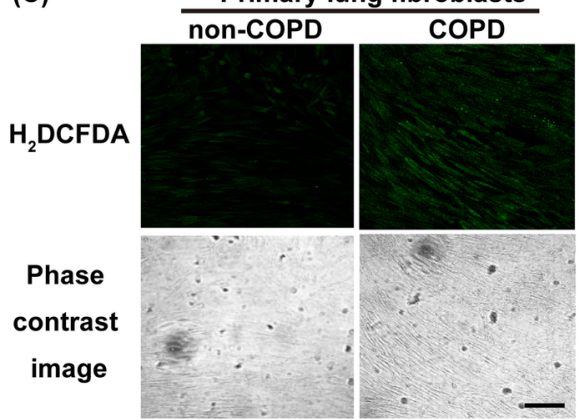

(E)

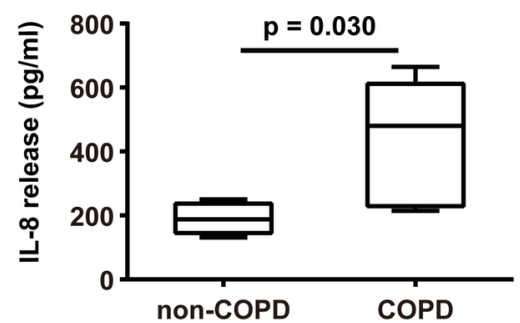

(G)

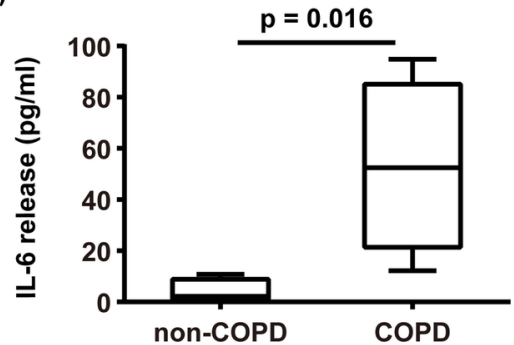

(B)

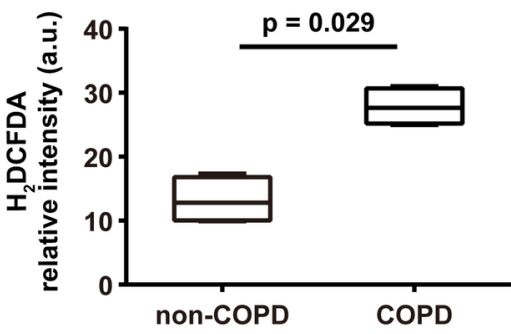

(D)

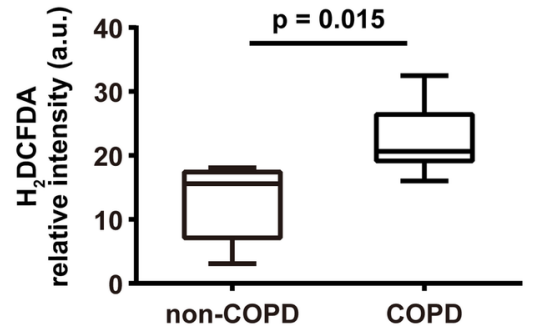

(F)

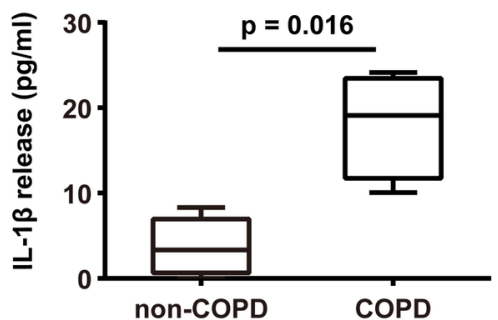

(H)

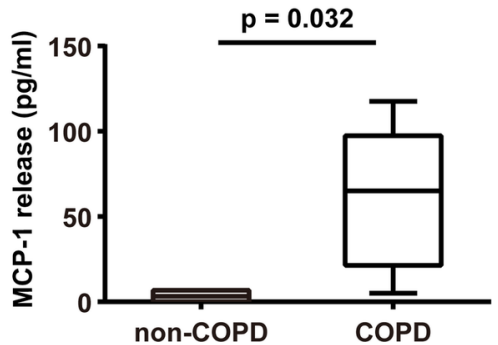

Figure 3 Production of reactive oxygen species (ROS), proinflammatory cytokines and chemokines from primary lung-resident cells. Primary bronchial epithelial cells from non-chronic obstructive pulmonary disease (COPD) subjects $(n=4)$ and patients with $\operatorname{COPD}(n=4)(A, B)$ and primary lung fibroblasts from non-COPD subjects $(n=6)$ and patients with COPD $(n=6)(C, D)$ were cultured and stained with 5-(and 6)-chloromethyl-2' $7^{\prime}$ dichlorohydrofluorescein diacetate (CM- $H_{2}$ DCFDA). The fluorescence intensities were measured using a fluorescence microplate reader. The amounts of interleukin (IL)-8 (E), IL-1 $\beta$ (F), IL-6 (G) and monocyte chemotactic protein (MCP)-1 (H) in the media of primary bronchial epithelial cells were measured using a cytometric bead array (IL-8: non-COPD, $n=6$; COPD, $n=5$; IL-1 $\beta$ : non-COPD, n=5; COPD, n=4; IL-6: non-COPD, $n=5$; COPD, $n=4$; MCP1: non-COPD, $n=5 ; C O P D, n=5$ ). Data are presented as the median (with interquartile ranges). Data were analysed using Wilcoxon rank sum tests. Scale bars $=100 \mu$ m. a.u., arbitrary units. 
(1.1-8.8) pg/mL, p=0.016; MCP-1: COPD, 65.0 (21.5-97.5) vs non-COPD, $3.3(0.0-6.8) \mathrm{pg} / \mathrm{mL}, \mathrm{p}=0.032)$.

\section{Decrease in extracellular reactive persulfide and polysulfide species in ELF from patients with COPD}

To quantify reactive persulfides and polysulfides on the surface of the respiratory tract, ELF samples obtained from the study subjects using the bronchoscopic microsampling method (see online supplementary table S1) were subjected to LC-ESI-MS/ MS analyses (figure 4 and see online supplementary figure S3). We identified GSSH (figure 4A), CysSSH (figure 4B), GSSSH (figure 4C), GSH (figure 4D) and CysSH (figure 4E) in the ELF, and the amounts of GSSH in the ELF samples from patients with COPD $\quad(37.0 \quad(24.5-49.0 \mathrm{nmol} / \mathrm{g}$ protein) were significantly decreased compared with levels from non-COPD subjects (68.5 $(45.3-97.5) \mathrm{nmol} / \mathrm{g}$ protein, $\mathrm{p}=0.004$, figure $4 \mathrm{~A})$. The levels of GSH in patients with COPD (8.2 (1.4-12.3) $\mu \mathrm{mol} / \mathrm{g}$ protein) were also significantly decreased compared with non-COPD subjects (22.2 (13.0-29.1) $\mu \mathrm{mol} / \mathrm{g}$ protein, $\mathrm{p}=0.002$, figure 4D), although the multiple regression analyses suggested that the measurements were significantly determined by sex, not the COPD disease status (see online supplementary Table S6). The amounts of GSSSH were also significantly decreased in the ELF samples from patients with COPD $(0.0(0.0-1.0) \mathrm{nmol} / \mathrm{g}$ protein) compared with amounts from non-COPD subjects $(2.0(1.0-6.5) \mathrm{nmol} / \mathrm{g}$ protein, $\mathrm{p}=0.004$, figure $4 \mathrm{C}$ ). There was no significant difference in the amounts of CysSSH and CysSH between the two groups (CysSSH: COPD, $11.0(6.5-14.5)$ vs non-COPD, $10.5(8.5-15.5) \mathrm{nmol} / \mathrm{g}$ protein, $\mathrm{p}=0.749$; CysSH: COPD, $1.0(0.4-1.3)$ vs non-COPD, 1.1 $(0.6-1.4) \mu \mathrm{mol} / \mathrm{g}$ protein, $\mathrm{p}=0.442)$.

\section{Increase in CBS and CSE immunoreactivities in the airways of} patients with COPD

We next investigated the expression of CBS and CSE in the lung tissues from study subjects (see online supplementary table S2). CBS and CSE immunoreactivities were mainly detected in the bronchial epithelium (figure 5A and B). The immunoreactivities of CBS were significantly increased in the bronchial epithelium of patients with COPD (39.0 (25.0-60.0) a.u.) compared with immunoreactivities from the non-COPD group of never-smokers (19.5 (8.5-25.0) a.u., $\mathrm{p}<0.001$, figure 5C) and from non-COPD ex-smokers (17.8 (13.0-25.3) a.u., $\mathrm{p}=0.006$, figure 5C). Similarly, the immunoreactivities of CSE were significantly increased in the bronchial epithelium of patients with COPD (30.5 (21.5-59.5) a.u.) compared with CSE levels in the non-COPD group of neversmokers (10.0 (3.0-14.3) a.u., $\mathrm{p}<0.001$, figure 5D) and from non-COPD ex-smokers (9.5 (3.0-13.0) a.u., $\mathrm{p}<0.001$, figure 5D). The value of $\mathrm{FEV}_{1}$ \%predicted was significantly correlated with

(B)

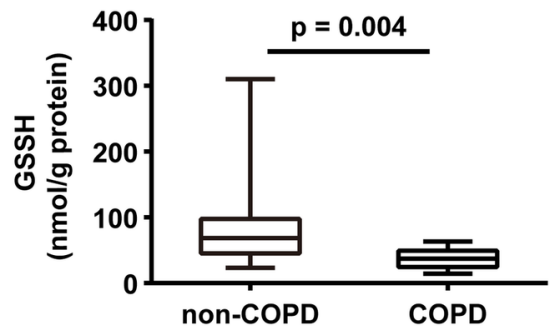

(C)

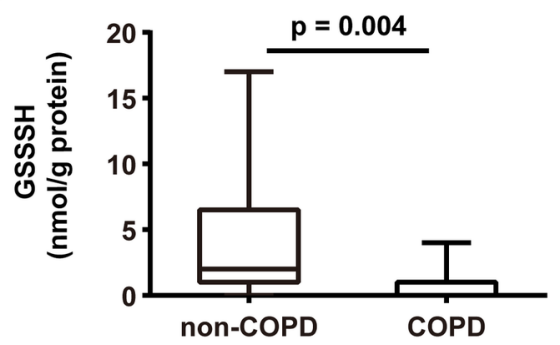

(D)
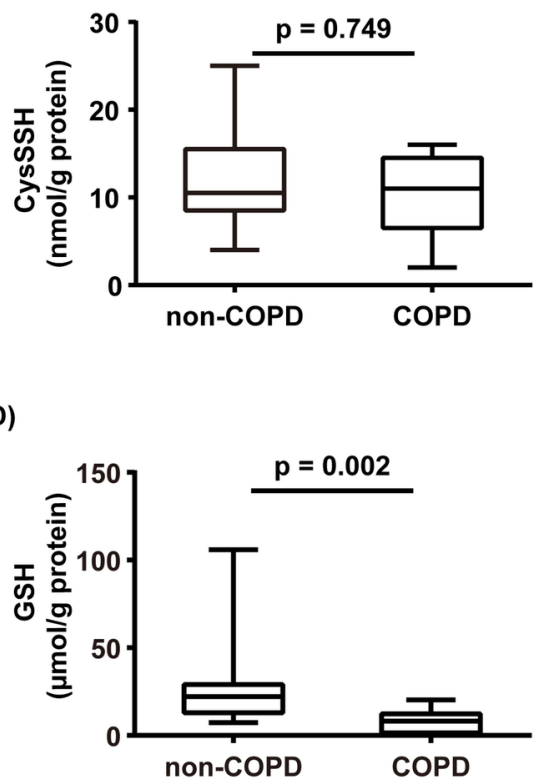

(E)

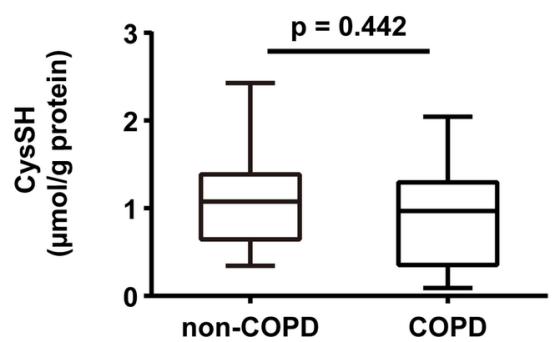

Figure 4 Determination of extracellular reactive persulfide and polysulfide species in epithelial lining fluid (ELF). ELF samples were obtained using the bronchoscopic microsampling method. ELF samples from 13 patients with COPD and 10 non-COPD subjects were subjected to LC-MS/MS analysis. Extracellular GSSH (A), CysSSH (B), glutathione trisulfide (GSSSH) (C), GSH (D) and CysSH (E) in ELF were identified. Data represent the median (with interquartile ranges). Data were analysed using Wilcoxon rank sum tests. 
the immunoreactivities of CBS $(\mathrm{r}=-0.29, \mathrm{p}<0.042$, figure $5 \mathrm{E})$ and CSE $(r=-0.37, p<0.009$, figure $5 F)$. To confirm the results in the immunohistochemical study, the amounts of CBS and CSE in primary lung-resident cells were examined by western blotting (see online supplementary figure S4). The amounts of CBS and CSE were significantly increased in the primary bronchial epithelial cells of patients with COPD compared with the amounts from non-COPD subjects (CBS, $\mathrm{p}=0.004$; CSE, $\mathrm{p}=0.004$ ) as well as those in primary lung fibroblasts (CBS, $p=0.008$; CSE, $\mathrm{p}=0.032$ ).
Increase in CBS and CSE in the sputum cells of patients with COPD

We further investigated the immunostaining of CBS and CSE in sputum cells. The characteristics of the subjects and the cell differentials in the sputum are shown in online supplementary tables S3 and S4, respectively. CBS and CSE immunoreactivities were mainly detected in macrophages and neutrophils in the sputum of patients with COPD (figure 6A and B). The percentages of CBS-immunopositive cells were significantly

(A)

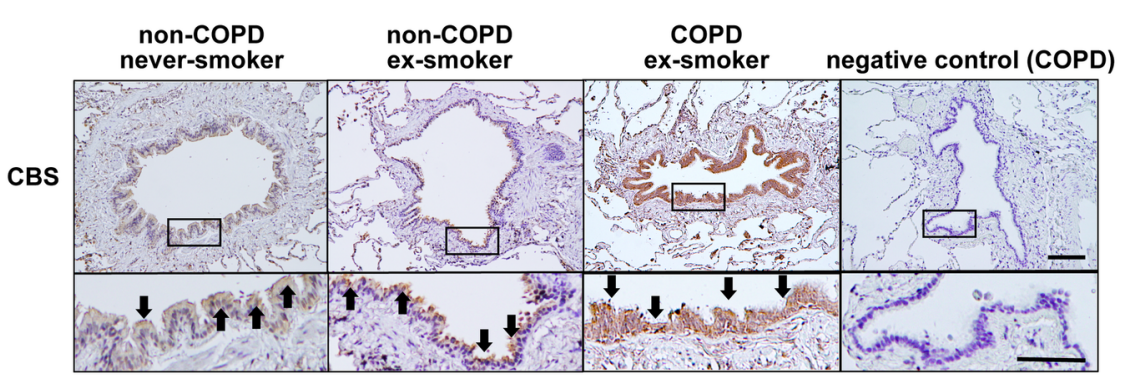

(B)

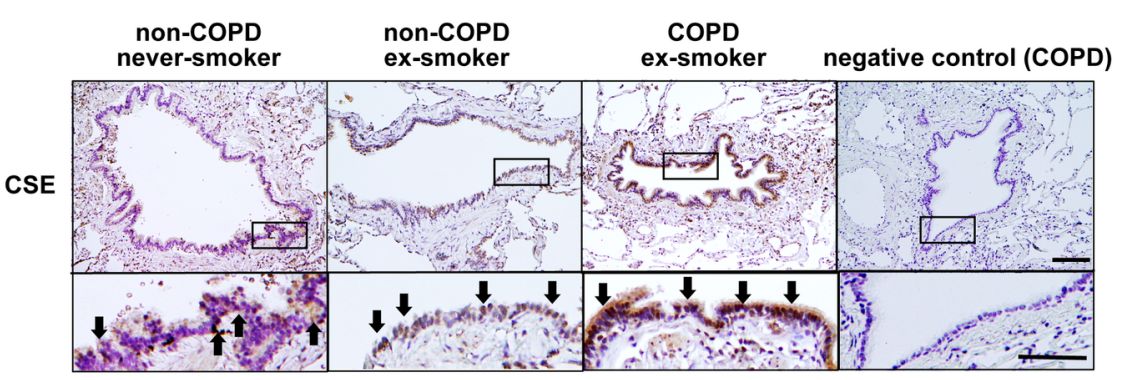

(C)

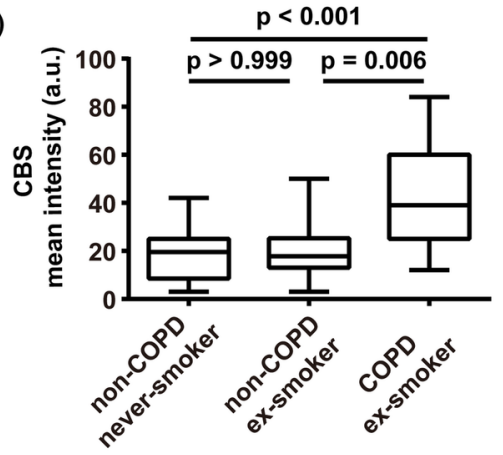

(E)

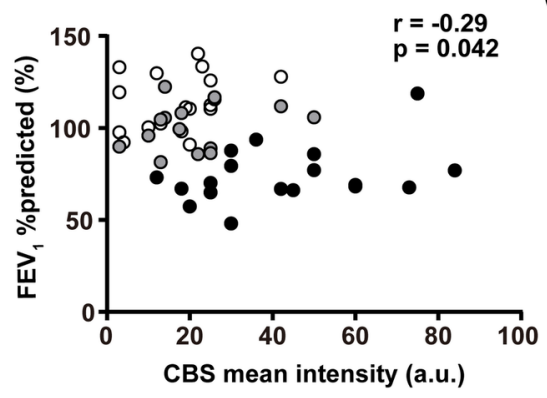

(D)

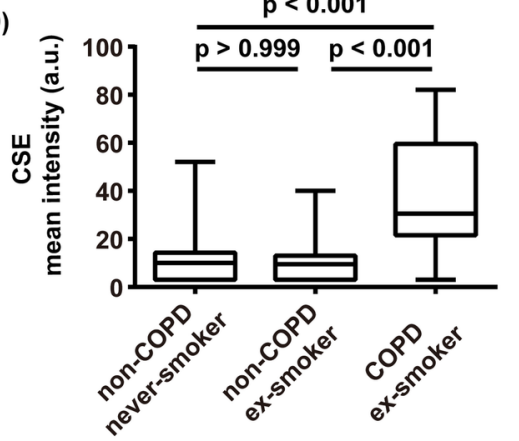

(F)

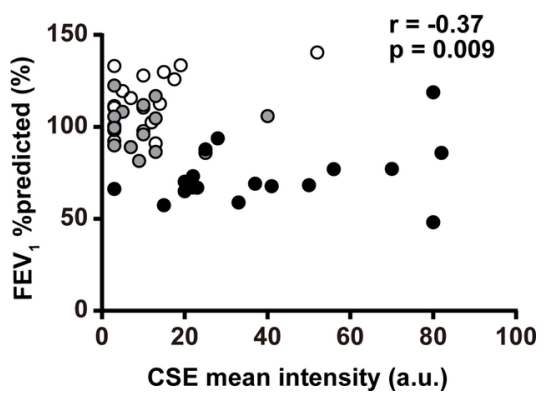

Figure 5 Localisation of cystathionine $\beta$-synthase (CBS) and cystathionine $\gamma$-lyase (CSE) in lungs. Representative photographs of immunohistochemical staining against CBS (A) and CSE (B) in the lung tissues are shown. Upper panels show low magnification and lower panels show higher magnification images. Arrows indicate immunopositive cells. CBS (C) and CSE immunoreactivities (D) in the bronchial epithelium were analysed by HistoQuest software with identical settings and were expressed as the mean intensities (a.u.). Correlations between the values of FEV \%predicted and the values of CBS (E) or CSE immunoreactivities (F) are shown. $r$ is the correlation coefficient. Data are presented as the median (with interquartile ranges). Data were analysed using a Kruskal-Wallis test followed by Dunn's test. Correlations were tested using Spearman's tests. White circles, non-COPD never-smokers; grey circles, non-COPD ex-smokers; black circles, COPD ex-smokers. Scale bars in upper panels=100 $\mu \mathrm{m}$, scale bars in lower panels $=50 \mu$ m. a.u., arbitrary units. 
increased in patients with COPD (7.1 (3.3-19.0)\%) compared with non-COPD subjects $(0.9(0.7-2.4) \%, \mathrm{p}<0.001$, figure $6 \mathrm{C})$. Similarly, the percentages of CSE-immunopositive cells were significantly increased in patients with COPD $(1.7(0.7-3.8) \%)$ compared with non-COPD subjects $(0.0(0.0-1.1) \%, \mathrm{p}=0.002$, figure 6D). The values of $\mathrm{FEV}_{1} \%$ predicted were significantly correlated with the percentages of CBS-immunopositive $(\mathrm{r}=-0.45, \mathrm{p}=0.005$, figure $6 \mathrm{E})$ and CSE-immunopositive cells $(\mathrm{r}=-0.40, \mathrm{p}=0.007$, figure $6 \mathrm{~F})$.

\section{DISCUSSION}

For the first time and by means of LC-MS/MS analysis, we demonstrated that the amounts of reactive persulfide and polysulfide species (including GSSH, CysSSH and GSSSH) were decreased in both the lung-resident cells and ELF obtained from patients with COPD. The production of reactive persulfides and polysulfides in the primary lung-resident cells was significantly correlated with the degree of airflow limitation of the study subjects. Thus, our findings suggest that reactive persulfide and polysulfide species, which are newly detected antioxidants, could be associated with the redox balance in lungs of patients with COPD.

Our previous reports demonstrated that $\mathrm{CysSSH}$ was synthesised from cystine in a process catalysed by CBS and CSE, which in turn may contribute to the generation of GSSH and polysulfide derivatives. ${ }^{14} 15$ The antioxidant capacity and reactivity

(A)

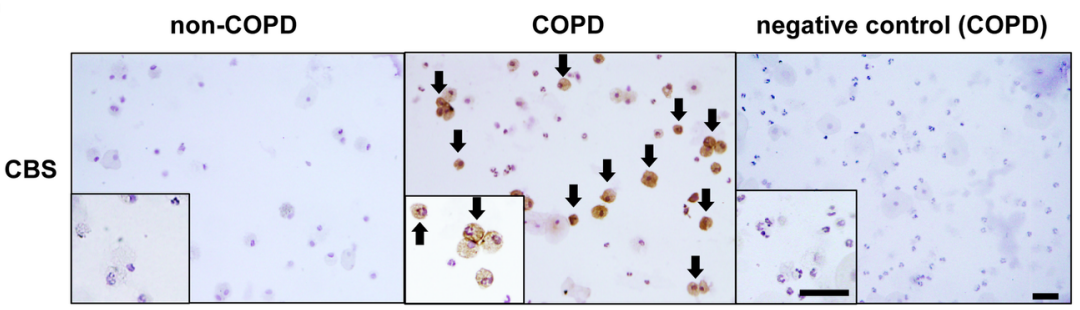

(B)

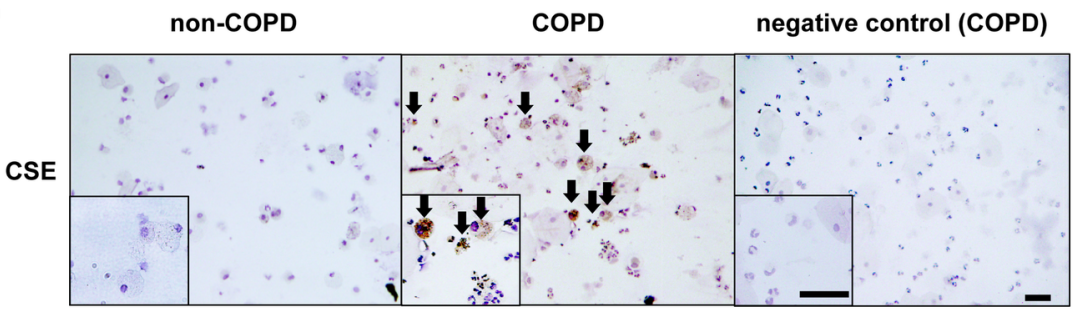

(C)

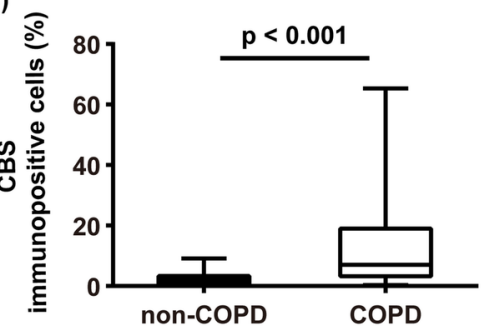

(E)

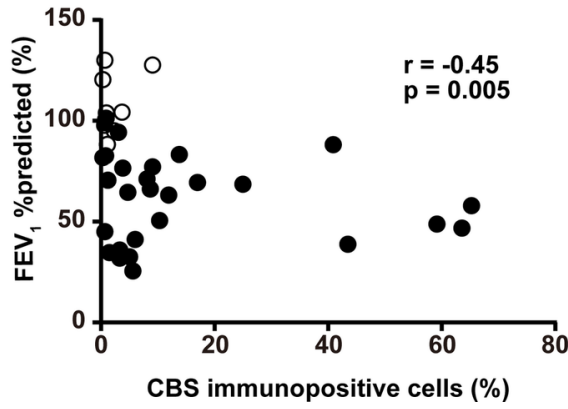

(D)

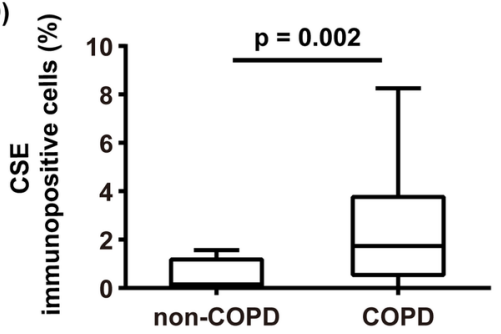

(F)

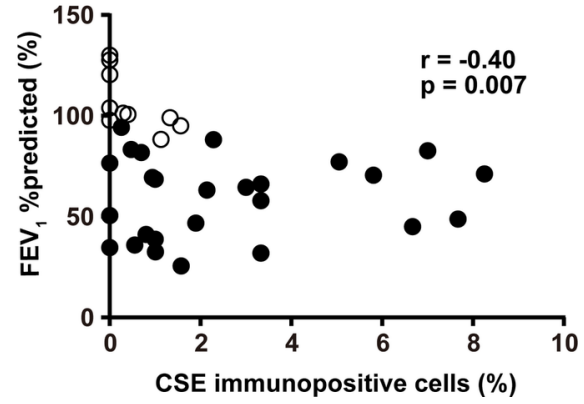

Figure 6 Detection of cystathionine $\beta$-synthase (CBS) and cystathionine $\gamma$-lyase (CSE) in sputum cells. Representative photographs of immunocytochemical staining against CBS (A) and CSE (B) are shown. Arrows indicate immunopositive cells. CBS (C) and CSE (D) immunopositive cells were counted and expressed as a percentage of total cells. Correlations between the values of FEV $\%$ predicted and the percentages of CBS (E) or CSE (F) immunopositive cells in sputum are shown. $r$ is the correlation coefficient. Data are presented as the median (with interquartile ranges). Data were analysed using a Wilcoxon rank sum test. Correlations were tested using Spearman's tests. White circles, non-COPD subjects; black circles, patients with COPD. Scale bars $=50 \mu \mathrm{m}$ (inset: scale bars $=25 \mu \mathrm{m}$ ). 
of GSSH and CysSSH are 10-100 times greater than those of GSH and CysSH. ${ }^{14} 151719$ This unique feature of reactive persulfides and polysulfides relies on adjacent electron pairs, an effect that is known as the $\alpha$ effect. ${ }^{15} 1719$ The importance of reactive persulfide and polysulfide species in the regulation of redox signalling is reinforced by the observation that these species exist in various organs of mice. ${ }^{14}{ }^{15}$ Moreover, there is increasing evidence to indicate that reactive persulfides and polysulfides actually regulate redox signalling mediated by various electrophilic oxidants. ${ }^{13-1623-25}$ To date, it has been believed that GSH plays a pivotal role in protecting cells against oxidative stress. ${ }^{310}$ However, the levels of GSH in previous studies were measured using a glutathione reductase recycling method ${ }^{3}{ }^{11}$, which is unable to detect reactive persulfide and polysulfide species. In addition, the antioxidant activity associated with GSH is typically mediated by specific enzymes such as GSH-dependent peroxidase. ${ }^{26}$ Without catalytic assistance, GSH is a relatively inert sulfhydryl with low nucleophilicity, and reacts poorly with electrophilic oxidants such as $\mathrm{H}_{2} \mathrm{O}_{2} \cdot{ }^{26}$ In this study, using the LC-MS/MS technique, we demonstrated that it was the amounts of intracellular GSSH and CysSSH rather than GSH or CysSH that were decreased in the lung cells of patients with COPD. These findings suggest that a decrease in GSSH and CysSSH could be associated with the state of redox imbalance in the cells from patients with COPD.

In the ELF, we observed that the levels of GSH-related metabolites rather than $\mathrm{CysSH}$-related metabolites were decreased in patients with COPD. The extracellular status of reactive persulfides and polysulfides may differ from the intracellular status. One possible explanation for this finding is that GSH-processing pathways are upregulated under inflammatory conditions. ${ }^{27}$ Gamma glutamyltransferase (GGT) is known to be involved in the breakdown of extracellular GSH and has also been described as having pro-oxidative properties. ${ }^{27}$ Given the complexities of the redox system in the lungs, further studies are necessary to clarify the various processes that are affected by the decrease in reactive persulfide and polysulfide species in the lungs of patients with COPD.

Contrary to our assumptions, we observed that the production of the synthases was enhanced in the lungs and airways of patients with COPD, even though the amounts of intracellular reactive persulfides and polysulfides were decreased. There are several possible mechanisms that explain this observation. First, oxidative stress may have a role. A previous study showed that treatment with $\mathrm{H}_{2} \mathrm{O}_{2}$ enhanced the production of CSE in A549 cells. ${ }^{28}$ Second, endoplasmic reticulum (ER) stress may have a role. It has been reported that ER stress occurs in the lungs of patients with COPD and could be involved in lung cell apoptosis and lung inflammation in COPD. ${ }^{29-31}$ When ER stress occurs, the transcription of CSE is upregulated through the protein kinase RNA-like ER kinase (PERK)-activating and eukaryotic translation initiation factor $2 \alpha$ (eIF $2 \alpha$ )-activating transcription factor 4 (ATF4) pathway. ${ }^{25} 32$ Taken together, the production of the synthases may be augmented in response to increased ER and oxidative stress in COPD.

We recognise that this study has limitations. First, the numbers of subjects were relatively small in parts of the analyses of our study due to the difficulty in obtaining enough primary lung cells or ELF samples, which may have resulted in insufficient power to detect significance for the study questions, though the $\mathrm{p}$ values indicated significant difference or correlation. Second, we could not investigate differences in all the variables among three groups (ie, non-COPD never-smokers, non-COPD ex-smokers and COPD ex-smokers). As shown in each table, there were significant differences among other factors including sex and smoking history between COPD and non-COPD subjects, which potentially influenced the results. However, multiple regression analyses indicated that other factors including sex and smoking history were not significant determinants, except in the case of GSH in ELF (figure 4D). The analyses also suggested that having COPD or not was a significant determinant or more powerful determinant than others in affecting the measured values (see online supplementary table S6). Third, we cannot exclude the influence of medications on the amounts of reactive persulfides and polysulfides and the expression of synthases. Future studies should investigate reactive persulfides and polysulfides (and the expression of the synthases) in treatment-naïve patients with COPD.

In conclusion, we demonstrated for the first time that the levels of intracellular and extracellular reactive persulfide and polysulfide species are decreased in the lungs of patients with COPD. We propose that reactive persulfides and polysulfides, which are newly detected antioxidants, could be associated with the redox balance in the lungs of patients with COPD.

Acknowledgements We thank Dr Satoshi Miyata (Department of Evidencebased Cardiovascular Medicine, Tohoku University Graduate School of Medicine) for performing the statistical analyses. We thank Mr Brent Bell for reading the manuscript.

Contributors TN: cell culture, biochemical studies, immunohistochemical analysis, interpretation of results. HS: design of the study, technical advice, interpretation of results, writing of the manuscript. TA: design of the study, technical advice, measurement of reactive persulfides, interpretation of results. TI: measurement of reactive persulfides, technical advice, interpretation of results. SF: technical advice, measurement of reactive persulfides, interpretation of results. AK: technical advice, interpretation of results, recruitment of patients. MY: technical advice, interpretation of results, recruitment of patients. KO: recruitment of patients, informed consent of patients, technical advice. YH: cell culture, biochemical studies, technical advice, interpretation of results. RT: recruitment of patients, informed consent of patients.KS: recruitment of patients, informed consent of patients. YS: cell culture, technical advice, interpretation of results. TH: biochemical studies, technical advice, interpretation of results. SY: recruitment of patients, technical advice, interpretation of results. NF: technical advice, interpretation of results. TO: recruitment of patients, informed consent of patients. TT: recruitment of patients, informed consent of patients. YH: recruitment of patients, informed consent of patients. YO: recruitment of patients, informed consent of patients.MI: design of the study, interpretation of results, writing of the manuscript.

Funding This study was supported by grants from the Japan Society for the Promotion of Science Grant (\#26293195, \#16K15453, \#16H05307, \#17H04180) and a grant from the Practical Research Project for Allergic Diseases and Immunology (Research on Allergic Diseases and Immunology) from the Japan Agency for Medical Research and Development, AMED.

Competing interests None declared.

Ethics approval Tohoku University Graduate School of Medicine.

Provenance and peer review Not commissioned; externally peer reviewed.

(c) Article author(s) (or their employer(s) unless otherwise stated in the text of the article) 2017. All rights reserved. No commercial use is permitted unless otherwise expressly granted.

\section{REFERENCES}

1 Global Initiative for Chronic Obstructive Pulmonary Disease (GOLD). Global strategy for the diagnosis, management and Prevention of COPD, Global Initiative for chronic Obstructive Lung Disease (GOLD). 2017 http://goldcopd.org.

2 Barnes PJ. New anti-inflammatory targets for chronic obstructive pulmonary disease. Nat Rev Drug Discov 2013;12:543-59.

3 Rahman I, Adcock IM. Oxidative stress and redox regulation of lung inflammation in COPD. Eur Respir J 2006;28:219-42.

4 Sugiura $\mathrm{H}$, Ichinose M. Nitrative stress in inflammatory lung diseases. Nitric Oxide 2011:25:138-44

5 MacNee W, Tuder RM. New paradigms in the pathogenesis of chronic obstructive pulmonary disease I. Proc Am Thorac Soc 2009;6:527-31.

6 Kirkham PA, Barnes PJ. Oxidative stress in COPD. Chest 2013;144:266-73. 
7 Dekhuijzen PN, Aben KK, Dekker I, et al. Increased exhalation of hydrogen peroxide in patients with stable and unstable chronic obstructive pulmonary disease. Am J Respir Crit Care Med 1996;154:813-6.

8 Montuschi P, Collins JV, Ciabattoni G, et al. Exhaled 8-isoprostane as an in vivo biomarker of lung oxidative stress in patients with COPD and healthy smokers. Am J Respir Crit Care Med 2000;162:1175-7.

9 Ichinose M, Sugiura H, Yamagata S, et al. Increase in reactive nitrogen species production in chronic obstructive pulmonary disease airways. Am J Respir Crit Care Med 2000;162:701-6.

10 Szabó C, Ischiropoulos H, Radi R. Peroxynitrite: biochemistry, pathophysiology and development of therapeutics. Nat Rev Drug Discov 2007;6:662-80.

11 Drost EM, Skwarski KM, Sauleda J, et al. Oxidative stress and airway inflammation in severe exacerbations of COPD. Thorax 2005;60:293-300.

12 van $\operatorname{der}$ Vliet $A, O^{\prime}$ Neill CA, Cross CE, et al. Determination of low-molecular-mass antioxidant concentrations in human respiratory tract lining fluids. Am J Physiol 1999;276:L289-96.

13 Nishida M, Sawa T, Kitajima N, et al. Hydrogen sulfide anion regulates redox signaling via electrophile sulfhydration. Nat Chem Biol 2012;8:714-24.

14 Ida T, Sawa T, Ihara H, et al. Reactive cysteine persulfides and S-polythiolation regulate oxidative stress and redox signaling. Proc Natl Acad Sci U S A 2014;111:7606-11.

15 Ono K, Akaike T, Sawa T, et al. Redox chemistry and chemical biology of H2S, hydropersulfides, and derived species: implications of their possible biological activity and utility. Free Radic Biol Med 2014;77:82-94.

16 Shinkai Y, Abiko Y, Ida T, et al. Reactive Sulfur Species-Mediated Activation of the Keap1-Nrf2 Pathway by 1,2-Naphthoquinone through Sulfenic Acids Formation under Oxidative Stress. Chem Res Toxicol 2015;28:838-47.

17 Saund SS, Sosa V, Henriquez S, et al. The chemical biology of hydropersulfides (RSSH): Chemical stability, reactivity and redox roles. Arch Biochem Biophys 2015;588:15-24

18 Millikin R, Bianco CL, White C, et al. The chemical biology of protein hydropersulfides: Studies of a possible protective function of biological hydropersulfide generation. Free Radic Biol Med 2016;97:136-47.

19 Cuevasanta E, Lange M, Bonanata J, et al. Reaction of hydrogen sulfide with disulfide and sulfenic acid to form the strongly nucleophilic persulfide. J Biol Chem 2015;290:26866-80.
20 Fulcher ML, Gabriel S, Burns KA, et al. Well-differentiated human airway epithelial cell cultures. Methods Mol Med 2005;107:183-206.

21 Togo S, Holz O, Liu X, et al. Lung fibroblast repair functions in patients with chronic obstructive pulmonary disease are altered by multiple mechanisms. Am J Respir Crit Care Med 2008; 178:248-60.

22 Ishizaka A, Watanabe M, Yamashita T, et al. New bronchoscopic microsample probe to measure the biochemical constituents in epithelial lining fluid of patients with acute respiratory distress syndrome. Crit Care Med 2001;29:896-8.

23 Greiner R, Pálinkás Z, Bäsell K, et al. Polysulfides link H2S to protein thiol oxidation. Antioxid Redox Signal 2013;19:1749-65.

24 Kimura H. Signaling molecules: hydrogen sulfide and polysulfide. Antioxid Redox Signal 2015;22:362-76.

25 Paul BD, Snyder $\mathrm{SH}$. $\mathrm{H}_{2}$ S signalling through protein sulfhydration and beyond. Nat Rev Mol Cell Biol 2012;13:499-507.

26 Hayes JD, Flanagan JU, Jowsey IR. Glutathione transferases. Annu Rev Pharmacol Toxicol 2005;45:51-88.

27 Dominici S, Paolicchi A, Corti A, et al. Prooxidant reactions promoted by soluble and cell-bound gamma-glutamyltransferase activity. Methods Enzymol 2005; 401:484-501.

28 Wang M, Guo Z, Wang S. Cystathionine gamma-lyase expression is regulated by exogenous hydrogen peroxide in the mammalian cells. Gene Expr 2012;15:235-41.

29 Steiling K, van den Berge M, Hijazi K, et al. A dynamic bronchial airway gene expression signature of chronic obstructive pulmonary disease and lung function impairment. Am J Respir Crit Care Med 2013;187:933-42.

30 Kelsen SG. The Unfolded Protein Response in Chronic Obstructive Pulmonary Disease. Ann Am Thorac Soc 2016;13 Suppl 2(Suppl 2):S138-45.

31 Hassan T, Carroll TP, Buckley PG, et al. miR-199a-5p silencing regulates the unfolded protein response in chronic obstructive pulmonary disease and $\alpha 1$-antitrypsin deficiency. Am J Respir Crit Care Med 2014;189:263-73.

32 Dickhout JG, Carlisle RE, Jerome DE, et al. Integrated stress response modulates cellular redox state via induction of cystathionine $\gamma$-lyase: cross-talk between integrated stress response and thiol metabolism. J Biol Chem 2012;287:7603-14. 